\title{
El sistema de riesgos laborales frente al trabajador del sector informal ${ }^{*}$
}

\author{
Juan Guillermo Ocampo** \\ María Osley Garzón*** \\ Recibido: septiembre de 2015 \\ Aprobado: octubre de 2016 \\ DOI: 10.22395/ojum.v15n30a9
}

\section{RESUMEN}

La Constitución Política considera el trabajo como un derecho fundamental dentro de la categoría de derecho social y económico, bajo los principios de igualdad, libertad y dignidad humana, y es el Estado el encargado de garantizar este derecho por medio del Sistema de Seguridad Social Integral que es un derecho irrenunciable para toda la población colombiana, en especial para el trabajador. El Departamento Administrativo Nacional de Estadística -DANE(DANE, 2015), en su informe de empleo del trimestre noviembre 2014-enero 2015, en 23 ciudades y áreas metropolitanas, determina que el $49.3 \%$ de la población laboral está en el sector informal. El objetivo del presente trabajo es poder establecer si para el trabajador del sector informal, principalmente el trabajador por subsistencia, existen elementos jurídicos desde la Constitución, la jurisprudencia y dentro de la legislación del Sistema General de Seguridad Social, para ingresar al Sistema de Riesgos Laborales.

Palabras clave: Seguridad social, sistema de riesgos laborales, trabajador informal, afiliación.

Este artículo se constituye en el trabajo de grado para optar el título de especialista en Salud Ocupacional, Universidad CES, 2015.

•* Autor principal. Médico y abogado, especialista en Gerencia de la Protección Social. Universidad CES. Correo electrónico: juanocampo7@gmail.com

** Co-autora y asesora metodológica. Administradora en Salud, Gestión Sanitaria y Ambiental, Magíster en Epidemiologia. Docente Facultad de Medicina Universidad CES-Medellín, Colombia. Correo electrónico: mgarzon@ces.edu.co. 


\section{The Labor Risk System before Workers of the Informal Sector}

\section{ABSTRACT}

The National Constitution has contemplated work as a basic right within the category of social and economic right, under the principles of equality, liberty, and human dignity. The State is the body responsible for assuring this right through the Integral Social Security System, which is an unalienable right for the entire Colombian population, especially for workers. The National Statistics Administrative Department (DANE, for its initials in Spanish) (DANE, 2015), in its employment report corresponding to the quarter between November 2014 and January 2015, involving 23 cities and metropolitan areas, determined that $49.3 \%$ of labor population is found in the informal sector. The objective of this article is to establish whether or not there are juridical elements (including Constitution, Juridical System, and the regulations of the General Social Security System) for the informal worker to be included in the Labor Risk System.

Key words: social security; labor risk system; informal worker; membership. 


\section{INTRODUCCIÓN}

La clasificación tradicional del trabajo dependiente-asalariado o independienteautoempleo, según la Organización Internacional del Trabajo (OIT) (Levaggi, 2004), ha llevado a diferenciarlo en trabajo formal con garantías laborales (incluyendo el acceso al Sistema de Protección Social), y trabajo informal sin estas garantías, situación que motiva la realización del presente artículo, como una reflexión alrededor de las desigualdades que pueden presentarse entre ambos tipos de trabajo, con el fin de ofrecer un punto de vista jurídico que podría aportar elementos que puedan ser soporte para cerrar esta brecha.

Reportes a 2015 del Departamento Administrativo Nacional de Estadística (DANE, 2015) informan que la población trabajadora del sector informal en el trimestre de noviembre 2014-enero 2015 en 23 ciudades y áreas metropolitanas fue de $49.3 \%$, similar al mismo trimestre del año anterior (DANE, 2014). Según este informe, el 92,3\% de los ocupados tenía acceso a seguridad social en salud de los cuales el 53,3\% pertenecía al régimen contributivo y de estos el $46,1 \%$ cotizaba a pensiones. No obstante, el informe no hace referencia a la afiliación al sistema de riesgos laborales y no especifica la inclusión o no de los trabajadores independientes que realizan actividades de subsistencial'. Este informe contrasta con

1 (Congreso de la República de Colombia, 2011). El empleo informal por subsistencia es definido por la Ley 1429 de 2010 como "el ejercicio de una actividad por fuera de los parámetros legalmente constituidos, por un individuo, familia o núcleo social para poder garantizar su mínimo vital" (Congreso de la República de Colombia, 2010). los datos publicados por Garzón (Garzón et al., 2014) en venteros ambulantes de la ciudad de Medellín, que presentaban un bajo nivel de afiliación al régimen de pensiones $(2 \%)$, una alta afiliación al régimen subsidiado en salud $(81,2 \%)$ y la inexistencia de afiliación a riesgo profesionales.

En Colombia, la Seguridad Social es un derecho irrenunciable de todo ciudadano que constituye una de las garantías mínimas del trabajo, definido en los artículos 48 y 53 de la Corte Constitucional (Colombia, 1991). Aunque estos derechos no necesariamente son garantistas del acceso al Subsistema de Riesgos Laborales para los trabajadores del sector informal, se ha legislado para permitir su vinculación a este Subsistema, situación que, según los reportes oficiales, aún no se ha materializado. Estos son aspectos que motivan la reflexión y dan pie a la realización del presente artículo, con el fin de aportar argumentos que permitan ahondar en el cuestionamiento de si el sistema de riesgos laborales en Colombia realmente ampara al trabajador informal.

Para dar respuesta, se definió el trabajo formal frente al informal, a partir de los conceptos de la OIT, haciendo énfasis en lo planteado en la XV y XVII Conferencia Internacional de Estadísticos del Trabajo (CIET), así como su desarrollo conceptual en Colombia desde diferentes estudios y planteamientos de la Corte Constitucional hasta llegar a la definición dada por el DANE. Posteriormente se realizó un estudio sistemático de los elementos jurídicos implementados en Colombia, desde el Plan Nacional de Salud Pública 
2007-2011, para permitir el ingreso del trabajador del sector informal al Sistema de Riesgos Laborales. Se efectuó el análisis a la Ley 1562 de 2012, que hace referencia a la afiliación de esta población laboral de manera voluntaria al Sistema de Riesgos Profesionales, planteando sus falencias y aciertos.

Se analizó el Decreto 2616 de 2013 que permite el acceso de un sector de los trabajadores del sector informal al sistema de riesgos laborales (Ministerio del Trabajo, 2013), en respuesta a uno de los objetivos del Plan Nacional de Desarrollo 2010-2014, que refiere la necesidad de diseñar un esquema financiero y operativo para posibilitar la afiliación del trabajador del sector informal por "subsistencia" a los sistemas de pensiones y de riesgos laborales (Congreso de la Republica de Colombia, 2011). Por último, el artículo concluye con una respuesta al interrogante planteado inicialmente con la que se pretende ofrecer una alternativa interpretativa al trabajador informal para poder acceder al Sistema de Riesgos Laborales.

\section{CONCEPTO DE TRABAJO INFORMAL}

\section{a. Según la 0IT}

El concepto de economía informal lo utilizo por primera vez la Organización Internacional del Trabajo -OIT- en 1972, basándose en el estudio realizado por Keith Hart en 1970 sobre los trabajadores urbanos de Ghana, donde definió al sector informal como "la fuerza de trabajo urbana no absorbida por el mercado de trabajo organizado" (Núñez Castrejón $\mathcal{E}$
Gómez Chiñas, 2008), que "al no ser incorporadas en los sectores más productivos de la economía deben desempeñarse en actividades de baja productividad y mal remunerados" (citado por Jaramillo, Gómez, \& García-Suaza, 2013). En 1993, la XV Conferencia Internacional de Estadísticos del Trabajo-CIET- de la OIT adoptó la resolución sobre las estadísticas del empleo en el sector informal y lo definió como:
Un conjunto de unidades dedi- cadas a la producción de bienes o la prestación de servicios con la finalidad primordial de crear empleos y generar ingresos para las personas que participan en esa actividad. Estas unidades funcionan típicamente en peque- ña escala, con una organización rudimentaria, en la que hay muy poca o ninguna distinción entre el trabajo y el capital como facto- res de producción. Las relaciones de empleo -en los casos en que existan- se basan más bien en el empleo ocasional, el parentesco o las relaciones personales y so- ciales, y no en acuerdos contrac- tuales que supongan garantías formales (OIT, 1993b).

Se creó una clasificación del empleo "según el tipo de contrato explícito o implícito de trabajo del titular con otras personas u organizaciones" (OIT, 1993a). Esta clasificación se fundamentó en criterios básicos como tipo de riesgo económico y tipo de autoridad que tendrán los titulares sobre los establecimientos y sobre otros trabajadores. Fue así como se derivaron los siguientes grupos: 1. Asalariados; 2. Empleadores; 3. Trabajadores por cuenta propia; 4. Miembros de cooperativas de 
productores; 5 . Trabajadores familiares auxiliares; 6 . Trabajadores que no pueden clasificarse según la situación en el empleo. En esta conferencia también se hizo una distinción entre empleos asalariados e independientes:

a. Empleos asalariados. Son "los titulares que tienen contratos de trabajo implícitos o explícitos (orales o escritos), por los que reciben una remuneración básica".

b. Empleos independientes: son aquellos donde la "remuneración depende directamente de los beneficios derivados de los bienes o servicios producidos" por ellos mismos o por medio de otros.

c. Trabajadores por cuenta propia: son quienes trabajan por sí mismo o con uno o más socios y se les define como empleo independiente y no han contratado a otras personas para que trabajen para ellos en forma continua.

También se definieron otros grupos particulares de trabajadores:

d. Trabajadores con empleos precarios: son los trabajadores cuyos contratos de trabajo se clasifican como:

- Trabajadores ocasionales: son los trabajadores que tienen un contrato de trabajo implícito o explícito de corta duración y los trabajadores pueden ser asalariados o por cuenta propia

- Trabajadores a corto plazo: son los trabajadores que tienen contratos de trabajo implícitos o explícitos con una duración prevista mayor a la establecida en cada nación para definir a los "trabajadores ocasionales", pero más corta que la utilizada para definir a los "asalariados regulares".

- Trabajadores estacionales: son los trabajadores que tienen contratos explícitos o implícitos de trabajo cuya duración y momento en que se efectúa dicho contrato están influenciados significativamente por factores estacionales tales como el ciclo del clima, los días festivos y/o las cosechas agrícolas.

e. Trabajadores de subsistencia: son los que tienen un "empleo independiente" y cuyo hogar consume la mayoría de los bienes o servicios producidos, constituyéndose una base importante para su subsistencia.

La XVII CIET (OIT, 2003) complementó la clasificación de empleo del sector informal de la XV CIET, al incluir los siguientes tipos de empleos:

a. Trabajadores por cuenta propia, dueños de sus propias empresas del sector informal;

b. Empleadores dueños de sus propias empresas del sector informal;

c. Trabajadores familiares auxiliares, independientemente que si trabajan en empresas del sector formal o informal;

d. Miembros de cooperativas de productores informales; 
e. Asalariados que tienen empleos informales, ya que estén empleados por empresas del sector formal, por empresas del sector informal, o por hogares que les emplean como trabajadores domésticos asalariados;

f. Trabajadores por cuenta propia que producen bienes exclusivamente para el propio uso final de su hogar.

Además, incluyó en este sector a los asalariados con una relación laboral no sujeta a "la legislación laboral nacional, el impuesto sobre la renta, la protección social o determinadas prestaciones relacionadas con el empleo (preaviso al despido, indemnización por despido, vacaciones anuales pagadas o licencia pagada por enfermedad, etc.)". De otro lado, Núñez Castrejón $\varepsilon$ Gómez Chiñas concluyen que el sector informal es "producto del desempleo, que se ha propagado a lo largo de muchos años, debido a causas estructurales de las economías menos desarrolladas (...) implementando tecnologías atrasadas que lejos de haber favorecido a los grupos más necesitados resultaron en un empeoramiento de sus condiciones de vida"(Núñez Castrejón \& Gómez Chiñas, 2008).

\section{b. Según el derecho colombiano}

Colombia desde la Constitución de 1991, se trasformó en un Estado social de derecho, convirtiendo el trabajo en principio, deber y derecho. En el preámbulo se le reconoce como un principio y en el artículo primero, junto con la dignidad humana, entre otros, se le reconoce como fundamento del Estado social de derecho, como lo sustenta la Corte Constitucional en su Sentencia T 581 de 1992:
El trabajo, como factor funda- mental de los procesos econó- micos y sociales, resulta de pri- mordial importancia en razón de que posibilita los medios de sub- sistencia y la calidad de ésta para el mayor número de la población y de él depende de manera ge- neral el crecimiento y desarrollo económico (Corte Constitucional de Colombia, 1992).

La Carta Magna expresa en su artículo 25 que "el trabajo es un derecho y una obligación social y goza, en todas sus modalidades, de la especial protección del Estado. Toda persona tiene derecho a un trabajo en condiciones dignas y justas"; y el artículo 26 determina que "toda persona es libre de escoger profesión y oficio..." (Colombia, 1991). Estos articulados se sustentan en la Declaración Universal de Derechos Humanos (ONU, n. d.), que estableció: 1. Toda persona tiene derecho al trabajo, a la libre elección de su trabajo, a condiciones equitativas y satisfactorias de trabajo y a la protección contra el desempleo. 2. Toda persona tiene derecho, sin discriminación alguna, a igual salario por trabajo igual. 3. Toda persona que trabaja tiene derecho a una remuneración equitativa y satisfactoria, que le asegure, así como a su familia, una existencia conforme a la dignidad humana y que será completada, en caso necesario, por cualesquiera otros medios de protección social. 
El Pacto Internacional de Derechos Económicos, Sociales y Culturales expresa que el derecho al trabajo "comprende el derecho de toda persona a tener la oportunidad de ganarse la vida mediante el trabajo libremente escogido o aceptado"(ONU, 1966). Es el Estado el llamado a garantizar el cumplimiento de estos principios como Estado social de derecho, que se fundamenta "en el respeto de la dignidad humana, en el trabajo y la solidaridad de las personas que la integran y en la prevalencia del interés general" (Colombia, 1991). El Ministerio de la Protección Social (Ministerio de Salud y Protección Social, n. d.), reafirma que el trabajo no puede estar aislado de la dignidad humana y, por ende, la responsabilidad del Estado "va más allá de garantizar el derecho a tener un trabajo (...) el empleado se encuentre en condiciones de seguridad, tranquilidad, estabilidad, igualdad, remuneración justa" y de esta forma se "garantice al trabajador la satisfacción más plena posible de todos sus derechos". Por lo tanto, el Ministerio del Trabajo acoge los objetivos estratégicos que plantea la OIT para el desarrollo del trabajo decente (OIT, 1996) los cuales propenden por 1. Promover y cumplir las normas y los principios y derechos fundamentales en el trabajo, 2. Crear mayores oportunidades para que mujeres y hombres puedan tener empleos e ingresos dignos, 3. Mejorar la cobertura y la eficacia de una seguridad social para todos, y 4. Fortalecer el tripartismo y el diálogo social.

La OIT plantea que la estructura tripartita ofrece "una plataforma desde la cual promover trabajo decente para todos los hombres y mujeres". Sus principales objetivos son: "fomentar los derechos laborales, ampliar las oportunidades de acceder a un empleo decente, mejorar la protección social y fortalecer el diálogo al abordar temas relacionados con el trabajo" (OIT, 1996). Estos principios tienen validez tanto para el trabajador del sector formal como para el del informal, pues el trabajo decente es fundamental para reducir la pobreza y es "un medio para lograr un desarrollo equitativo, inclusivo y sostenible" (Ministerio de Salud y Protección Social, n. d.). Según Farné, Vergara, E Baquero, el Ministerio de la Protección Social no ha logrado grandes avances, al concluir que los resultados han sido decepcionantes y que las políticas social y laboral "no han podido magnificar los beneficios derivables de la mayor riqueza y productividad del país y no han permitido mejorar en la extensión deseada las condiciones cualitativas promedio de desempeño y bienestar de la fuerza de trabajo" (Farné, Vergara, E Baquero, 2011).

En el desarrollo de la clasificación del trabajo del sector formal frente al informal, se han adelantado diferentes estudios como el de Núñez Méndez, quien concibe al sector informal como:

Un trabajo no declarado, no protegido, no cubierto por la seguridad social ni pagado bajo las leyes del salario mínimo, e igualmente, es aquel al que se le facilita la evasión y el fraude de la seguridad social, el impuesto a la renta y el IVA (...) las condiciones 
de trabajo del sector informal son inferiores a las del sector formal en cuanto a higiene y condiciones de salud y seguridad (Núñez Méndez, 2002).

Igualmente caracteriza al trabajador del sector informal bajo tres aspectos: 1 . El trabajo informal no está cubierto por la seguridad social y no es remunerado bajo las leyes del salario mínimo; 2. Esta actividad es desarrollada por grupos marginados como desempleados, individuos de bajos ingresos e inmigrantes; y 3. Los trabajadores del sector informal se enfrentan a condiciones de higiene y salud inferiores a las de los trabajadores del sector formal. Flórez (2002) indica que la clasificación dada por el DANE es inadecuada, pues no reconoce la heterogeneidad del sector informal y considera como único elemento distintivo entre el sector formal e informal la afiliación o no al subsistema de salud; así deja de lado la afiliación a pensiones y el tener un salario mínimo o una jornada laboral máxima según la normativa definida por el Gobierno, por lo que plantea dividirlo en tres subsectores de ocupados informales: subsistencia directa, asalariados informales y empresarios informales. Por su parte, Ribero plantea cuatro definiciones para el sector informal (citado por Guataquí R, García S, E Rodríguez A, 2011):

1. Trabajadores independientes. Excluye a los profesionales independientes.

2. La definición 1, más los trabajadores familiares sin remuneración y los empleados domésticos.
3. Empleados en firmas con 10 o menos trabajadores.

4. Definición radical: trabajadores que no tienen seguridad social ni en salud, ni en pensiones, ni tienen un contrato laboral formal.

Guataqui y colaboradores analizan otros estudios como el de Ortiz y Uribe, García y Mondragón-Vélez et al., quienes llegan a conclusiones similares sobre el empleo del sector informal: tener unos ingresos más bajos y poca afiliación a la seguridad social. Cada estudio plantea definiciones similares sobre el concepto de empleo informal, el cual es un concepto dinámico y que puede obedecer a la realidad del mercado laboral en su momento. Consideran empleo formal si: pertenece al régimen contributivo o especial de salud, como cotizante y no como beneficiario; y estar cotizando a un fondo de pensiones o estar pensionado. Se considera empleo informal si no cumple alguno de los criterios mencionados, en especial si no están cotizando a salud.

El DANE caracteriza como empleo informal si son: empleados particulares y obreros que laboran en establecimientos, negocios o empresas que ocupen hasta cinco personas en todas sus agencias y sucursales, incluyendo al patrono y/o socio; trabajadores familiares sin remuneración; trabajadores sin remuneración en empresas o negocios de otros hogares; empleados domésticos; jornaleros o peones; trabajadores por cuenta propia que laboran en establecimientos de hasta cinco personas, excepto los independien- 
tes profesionales; los patrones o empleadores en empresas de cinco trabajadores o menos. Se excluyen los obreros o empleados del Gobierno (DANE, 2009). Esta caracterización se basa en la resolución 15. ${ }^{\text {a }}$ de la OIT de 1993 y en las recomendaciones dadas por el grupo de Delhi ${ }^{2}$ en el 2003 (Vásquez-Trespalacios \& MartínezHerrera, 2013).

Siendo el trabajo un derecho fundamental dentro de la categoría de derecho social y económico bajo los principios de igualdad, libertad y dignidad humana, la Corte Constitucional lo reafirma bajo tres dimensiones, sin distinción entre formalidad o informalidad:

1. El trabajo como garantía de las condiciones mínimas de subsistencia, como medio para la atención del mínimo vital. Cada individuo tiene la libertad de desarrollar una actividad legítima para obtener los recursos necesarios para cubrir sus necesidades personales y familiares en condiciones dignas y justas.

2. El trabajo puede concebirse como un presupuesto de la autonomía personal. Si bien en situaciones extremas, el mínimo vital puede satisfacerse a través de redes de apoyo familiar, social o, incluso, estatal, por medio de la asistencia pública o subsidios al desempleo, ello, sin embargo,

2 El Grupo de Expertos sobre estadísticas del sector no estructurado, comúnmente denominado "Grupo de Delhi", se creó en 1997 en el marco de los grupos de las ciudades de la Comisión de Estadística con el fin de abordar diversos asuntos metodológicos relativos al tratamiento del sector no estructurado. no satisface la aspiración de autonomía, que es condición misma de la dignidad de la persona.

3. El trabajo es también una condición de la realización personal, que permita desarrollar sus capacidades y potencialidades, sentirse útil y obtener reconocimiento social. Además, no se puede pasar por alto que la sociedad se construye con el esfuerzo de la misma comunidad a través de su participación social y laboral (Mendoza Martelo, 2010).

Según reportes del DANE respecto al empleo formal e informal en el área urbana en las 23 principales áreas metropolitanas entre 2001-2010, la informalidad ha representado aproximadamente un 50\%, una tendencia que va en ascenso, como lo describe la revista Portafolio.com, que para junio de 2011, el 65\% de las personas ocupadas estaban por fuera del sistema de riesgos profesionales, haciendo referencia a los trabajadores del sector informal, afirmando que están en un estado de "total desprotección en materia de accidentes y enfermedades laborales" por parte del Subsistema de Protección laboral; estas personas, al presentar un accidente laboral o una enfermedad derivada de su trabajo, son atendidas con recursos del sistema de salud, generalmente del régimen subsidiado (Portafolio, 2011). Esta realidad es más evidente en el estudio de Garzón, sobre un grupo de trabajadores del sector informal "venteros" del centro de Medellín, donde se determinó que el 
"93,5\% de los "venteros" estaba afiliado a salud, $81,2 \%$ era del régimen subsidiado y $12,4 \%$ como beneficiarios en el régimen contributivo; el porcentaje restante aparecía como vinculado; solo el 1,8\% cotizaba a pensiones y ninguno estaba afiliado a algún sistema de Riesgos laborales. (Garzón Duque et al., 2014). Estos datos concuerdan con los presentados por la Dirección Seccional de Salud de Antioquia en un análisis de salud del Departamento, 2012, al plantear que:

La situación que se presenta específicamente en Colombia, tiene que ver más con la accidentalidad laboral de la población vulnerable, la cual en su inmensa mayoría pertenece al régimen subsidiado de seguridad social y cuando esta población sufre un accidente de trabajo en el mejor de los casos, su atención hospitalaria se configura como enfermedad común, quedando en esta variable oculta toda la accidentalidad del sector vulnerable (Dirección Seccional de Salud de Antioquia, 2013).

Además, afirma que "este tipo de trabajadores no asistan en busca de atención médica para el cuidado de problemas que consideran menores, dado que si dejan de trabajar un día ponen en riesgo su supervivencia y la de las personas que tienen a su cargo". Igualmente plantean que el modelo económico colombiano llevará a un mayor incremento de la población laboral informal en los próximos años realizando, entre otros oficios, las ventas ambulantes en las grandes ciudades como consecuencia del desempleo, el subempleo, la violencia y el desplazamiento forzoso, como un medio para garantizar su subsistencia y el de su núcleo familiar. Una de las críticas que plantea es que esta población ha sido poco intervenida, entre otras razones, porque la visión de la salud ocupacional "se ha enfocado más hacia la formalidad en la gran empresa; las pequeñas y medianas empresas no han tenido hasta ahora un desarrollo suficiente". Además, "la población vulnerable, por su parte, no puede iniciar labores de salud ocupacional de una manera autónoma y sin orientaciones que, a su vez, tengan el sustento y soporte del sector académico y del gubernamental".

Podría decirse que en Colombia, el trabajo informal no ha tenido una definición completa, pues no especifica los empleos que no tienen contrato ni beneficios laborales, entre otros (Bustamante Izquierdo, 2011). En general, los trabajadores del sector informal se caracterizan por tener baja productividad y trabajos precarios ${ }^{3}$, así como por la baja cobertura en seguridad social en salud, pensiones y riesgos laborales. "La ausencia de protección social es una característica que define a las personas ocupadas en sector informal" (VásquezTrespalacios \& Martínez-Herrera, 2013). Esta situación es conocida por el Ministerio de Hacienda al determinar que la informalidad laboral es "un reflejo de

3 El trabajo precario se caracteriza, según Mariana Barattini (Barattini, 2009), el que cumple con alguno de estas condiciones:

- Empleos a plazo fijo (empleo temporario), eventuales, por subcontrato o a domicilio, el empleo a tiempo parcial

- El empleo clandestino o no registrado

- El empleo asalariado fraudulentamente oculto bajo formas no laborales 
la calidad del empleo en el país pues se caracteriza por bajos niveles de cobertura en seguridad social, bajos niveles de educación, e ingresos menores que los del empleo informal" (Bustamante Izquierdo, 2011). Esta desprotección en términos de seguridad social de los trabajadores del sector informal se ve reflejada en que solo el $10.1 \%$ de estos trabajadores están afiliados a salud y pensiones, pero al analizar por separado las tasas de afiliación, se "encuentra que la proporción de informales con seguridad social en salud es del $84.6 \%$ mientras que en pensiones corresponde solo al 10.3\%" (Bustamante Izquierdo, 2011).

Esta situación no es ajena a Colombia, pues en estudios similares en otros países latinoamericanos se concluye que la afiliación a los sistemas de seguridad social es muy baja, a pesar de las políticas implantadas para la protección de los trabajadores informales.

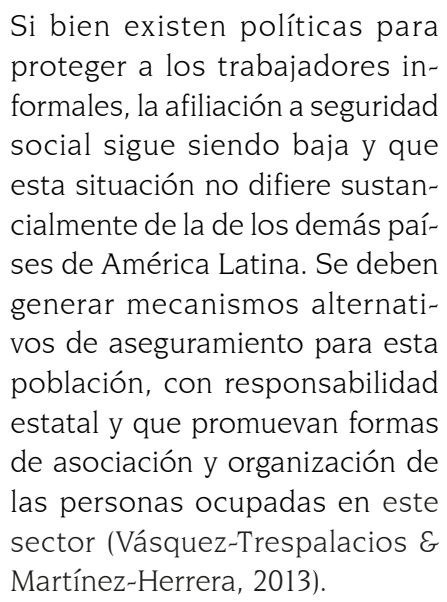

En términos generales, el empleo informal en Colombia no ha estado en el cen- tro del debate político y normativo para dignificarlo, en búsqueda de alternativas tales que garanticen el acceso al Sistema General de Seguridad Social en pleno (salud, pensión y riesgos profesionales), principalmente a quienes tienen empleos de subsistencia dentro de la informalidad.

\section{LA PROTECCIÓN DEL TRABAJADOR DEL SECTOR INFORMAL FRENTE A RIESGOS LABORALES EN LA LEY, LA JURISPRUDENCIA Y LA DOCTRINA}

El Ministerio de la Protección Social, por medio del Decreto 3039 de 2007 (Ministerio de la Protección Social., 2007), adoptó el Plan Nacional de Salud Publica 20072011, estableciendo dentro del eje de riesgos laborales, planes de acción de promoción y prevención y desarrollo de estudios de caracterización de las condiciones de salud y trabajo de la población laboral vulnerable. El Ministerio de Trabajo, a finales del 2012, manifestó que estaban desarrollando mecanismos de protección en cobertura de riesgos laborales para el trabajador del sector informal (Ministerio del Trabajo, 2012) y el Ministerio de Salud y Protección Social desarrollaba el Plan decenal de Salud Publica 2012-2021 por medio de la Resolución 1841 de 2013, que considera un conjunto de políticas e intervenciones que buscan el bienestar y protección de la salud de los trabajadores del sector formal e informal de la economía, ampliando la cobertura en el Sistema de Riesgos Laborales y promoviendo la promoción de la salud y la prevención de los riesgos laborales y ocupacionales por medio de dos componentes: a. Seguridad y salud en el trabajo. b. Situaciones pre- 
valentes de origen laboral (Ministerio de Salud y Protección Social, 2013a).

Bajo el primer componente, se plantea facilitar el acceso al Sistema General de Riesgos Laborales con base en la Ley 1562 de 2012 (Congreso de la Republica de Colombia, 2012), con el fin de lograr para el 2021, realizar las acciones de promoción de la salud y prevención de riesgos laborales en la población del sector informal, además que para este mismo periodo se logre que el 100\% "de las entidades territoriales gestionen la inclusión del componente de salud y ámbito laboral en los planes de desarrollo territorial y en los de ordenamiento territorial, en coordinación con las autoridades territoriales, departamentales, distritales y municipales" (Ministerio de Salud y Protección Social, 2012). El segundo componente va dirigido a disminuir los accidentes de trabajo y a evidenciar las enfermedades laborales en el proceso de calificación de origen y pérdida de la capacidad laboral en las diferentes instancias. Una de las estrategias planteadas es fomentar la vigilancia epidemiológica ocupacional a través de los entes territoriales de salud para planear las intervenciones necesarias, priorizando las siguientes actividades del sector informal: agricultura; ganadería; silvicultura y pesca; manufactura; minería; comercio; artesanos y manufactura.

El Ministerio de Salud y Protección Social ha expresado que

[...] el Sistema General de Riesgos Laborales, tiene como propósito: prevenir, proteger y atender a los trabajadores frente a las contingencias generadas por las enfermedades laborales y los accidentes de trabajo, que puedan ocurrir con ocasión o como consecuencia del trabajo (Ministerio de Salud y Protección Social, 2013b).

Como se puede deducir, no hace distinción entre trabajo formal e informal. Para febrero de 2014, el Ministerio de Trabajo publicaba una cartilla de riesgos laborales, dando a conocer los derechos y deberes que tienen tanto empleadores como trabajadores en el Sistema General de Riesgos Laborales. Afirma la cartilla que el trabajador independiente y del sector informal podrán acceder a este sistema, siempre y cuando coticen al régimen contributivo en salud (Ministerio del Trabajo, 2014).

El Sistema General de Riesgos Laborales hace parte del Sistema General de Seguridad Social, y la Ley 1562 de 2012 lo define como un "conjunto de entidades públicas y privadas, normas y procedimientos, destinados a prevenir, proteger y atender a los trabajadores de los efectos de las enfermedades y los accidentes que puedan ocurrirles con ocasión o como consecuencia del trabajo que desarrollan." Además, aclara quiénes deben afiliarse al Sistema General de Riesgos Laborales, en forma obligatoria y voluntaria.

\section{1) En forma obligatoria:}

a. Los trabajadores dependientes nacionales o extranjeros, vinculados mediante contrato de trabajo 
escrito o verbal y los servidores públicos; las personas vinculadas a través de un contrato formal de prestación de servicios con entidades o instituciones públicas o privadas [...] con una duración superior a un mes.

b. Las cooperativas y pre cooperativas de trabajo asociado son responsables, conforme a la ley, del proceso de afiliación y pago de los aportes de los trabajadores asociados;

c. Los jubilados o pensionados, que se reincorporen a la fuerza laboral como trabajadores dependientes, vinculados mediante contrato de trabajo o como servidores públicos;

d. Los estudiantes de todos los niveles académicos de instituciones educativas públicas o privadas que deban ejecutar trabajos que signifiquen fuente de ingreso para la respectiva institución o cuyo entrenamiento o actividad formativa es requisito para la culminación de sus estudios, e involucra un riesgo ocupacional.

e. Los trabajadores independientes que laboren en actividades catalogadas por el Ministerio de Trabajo como de alto riesgo. El pago de esta afiliación será por cuenta del contratante.

f. Los miembros de las agremiaciones o asociaciones cuyos trabajos signifiquen fuente de ingreso para la institución.

g. Los miembros activos del Subsistema Nacional de primera respuesta y el pago de la afiliación será a cargo del Ministerio del Interior, de conformidad con la normatividad pertinente.

2) En forma voluntaria: Los trabajadores independientes y los informales, diferentes de los establecidos en el literal a del presente artículo, podrán cotizar al Sistema de Riegos Laborales, siempre y cuando coticen también al régimen contributivo en salud y de conformidad con la reglamentación que para tal efecto expida el Ministerio de Salud y Protección Social en coordinación con el Ministerio del Trabajo en la que se establecerá el valor de la cotización según el tipo de riesgo laboral al que está expuesta esta población.

El Decreto 723 de 2013 (Ministerio de Trabajo, 2013) reglamenta la afiliación de las personas vinculadas a través de un contrato formal de prestación de servicios con entidades o instituciones públicas o privadas y de los trabajadores independientes que laboren en actividades de alto riesgo (clase IV y V), definidos en el artículo 26 del Decreto 1295 de 1994 (Ministerio de Trabajo, 1994), y clasificados por actividades económicas establecidas en el Decreto 1607 de 2002 (Ministerio de Trabajo, 2002). Como se observa, la Ley 1562 de 2012 y el Decreto 723 de 2013 no consideraron al trabajador del sector informal que no realiza ninguna actividad 
catalogada como de alto riesgo, o que no tiene un contrato formal superior a un mes, o que no cotiza al régimen contributivo en salud simplemente porque su empleo es de subsistencia, dejando por fuera y sin protección en riesgos laborales a una gran población trabajadora, pues como lo expresa Gómez Peñaloza, la mayoría de los trabajadores del sector informal no cuentan con la "solvencia suficiente para trasladar dicho riesgo a las Aseguradoras de Riesgos Laborales (ARL), mediante el pago de una cotización, para buscar de esta forma, asegurarse frente a las contingencias que puedan surgir en el trabajo tales como enfermedades profesionales, invalidez o muerte" (Gómez Peñaloza, 2014).

Aunque la normativa no desarrolló el tema de afiliación para este sector de la informalidad, sí lo ha desarrollado la jurisprudencia en torno a la responsabilidad del Sistema General de Riesgos Laborales de proteger a esta población, al pronunciarse la Corte Constitucional el sentido de que la seguridad social es un derecho fundamental, y que "la protección constitucional en materia de riesgos profesionales se garantiza asegurando el derecho irrenunciable a la seguridad social (Corte Constitucional de Colombia, 2004)". Además, determina que "basta al beneficiario de las prestaciones que de ella se desprenden, acreditar el vínculo laboral y la realización del riesgo con ocasión o como consecuencia del trabajo" (Corte Suprema de Justicia, 2005), según lo argumentado por Gómez Peñaloza:

Esta afirmación de la Corte Suprema (Sentencia 22656 de junio
30 de 2005), es determinante para poder concluir que a los trabajadores independientes a los que se les está negando su afiliación por carecer de un contrato superior a un mes con las formalidades indicadas atrás (Decreto 723 de 2013), pueda ser sujeto de protección por el Sistema de Riesgos Laborales, pues en el momento que a dicho afiliado le sobrevenga la enfermedad o accidente realizando las labores para las cual fue contratado, deberá ser quien tenga la responsabilidad de acreditar que ese riesgo fue con ocasión o causa del trabajo, luego bajo el entendido de la responsabilidad objetiva del sistema de Riesgos Labores, las administradoras no tendrán como argumento para negar la afiliación de estos trabajadores independientes, el hecho que el trabajador no tenga vigilados los riesgos y de esta forma sea indefinible si aquellos fueron de origen común o se crearon con causa u ocasión del trabajo, ya que es el beneficiario de las prestaciones quien debe acreditar aquello (letra cursiva subrayada fuera de texto) (Gómez Peñaloza, 2014).

La producción normativa en materia de riesgos laborales a la luz de los principios de la seguridad social como los de igualdad, progresividad y universalidad, para los trabajadores independientes y del sector informal (grueso de la población ocupada colombiana), ha sido insuficiente, pues históricamente han sido rezagados de cualquier política protectora en materia de riesgos profesionales ya que, lejos de ser incluyente, generó limitantes en muchos de ellos que, aun teniendo 
la solvencia para ingresar al sistema de riesgos laborales, no podrán afiliarse por carecer de un contrato formal, entre otros requisitos. Igualmente dejó al margen a los que trabajan por cuenta propia y a trabajadores con empleos de subsistencia, quienes no tendrían cómo configurar la relación de prestación del servicio a un tercero.

\section{ANÁLISIS DEL DECRETO 2616 DE 2013}

Teniendo presente que la Ley 1562 de 2012 y el Decreto 723 de 2013 no consideraron al trabajador del sector informal que no realiza ninguna actividad catalogada como de alto riesgo, que no tiene un contrato formal superior a un mes para su afiliación a riesgos laborales, o no pueda cotizar a salud, el Ministerio del Trabajo emitió el Decreto 2616 de 2013 (Ministerio del Trabajo, 2013) conocido como el Decreto de las empleadas domésticas por días, buscando llenar este vacío normativo con el objetivo de formalizar un sector de la población laboral informal y brindarle los beneficios del Sistema General de Seguridad Social y de Riesgos Laborales por medio de la vinculación y cotización a los sistemas de pensiones, riesgos laborales y subsidio familiar. Para acceder a estos beneficios, el trabajador del sector informal debe cumplir con las siguientes condiciones: a) que se encuentre vinculado laboralmente; b) que el contrato sea a tiempo parcial, es decir, que en un mismo mes, sea contratado por periodos inferiores a treinta (30) días, y c) que el valor que resulte como remuneración en el mes, sea inferior a un (1) salario mínimo mensual legal vigente.
Esta medida pretende dar cumplimiento al Plan nacional de desarrollo 2010-2014, establecido en la Ley 1450 de 2011(Congreso de la República de Colombia, 2011), en lo referente a formalización del empleo y reducir los índices de pobreza por medio de mecanismos como la vinculación a los sistemas de pensiones y riesgos laborales a los trabajadores del sector informal por subsistencia, definidos por la Ley 1429 de 2010 como "aquella [población] que se caracteriza por el ejercicio de una actividad por fuera de los parámetros legalmente constituidos, por un individuo, familia o núcleo social para poder garantizar su mínimo vital" (Congreso de la Republica de Colombia, 2010), y cumplir la Ley 100 de 1993, que establece la necesidad de garantizar la vinculación al Sistema General de Seguridad Social (Congreso de la Republica de Colombia, 1993). Pero este Decreto dejo nuevamente por fuera a la población laboral informal que no está vinculada a un contrato, independiente de si gana más o menos un salario mínimo mensual, o que labore más o menos de la jornada máxima permitida. El Decreto 1443 de 2014 dicta las disposiciones para la implementación del sistema de gestión de la seguridad y salud en el trabajo, pero esta norma tampoco tiene en cuenta al trabajador independiente (Ministerio de Trabajo, 2014).

\section{CONCLUSIÓN: ¿EL SISTEMA DE RIESGOS LABORALES EN COLOMBIA REALMENTE AMPARA AL TRABAJADOR INFORMAL?}

Nuestra Constitución ha determinado que el trabajo "es un derecho y una obligación social y goza, en todas sus modalidades, 
de la especial protección del Estado. Toda persona tiene derecho a un trabajo en condiciones dignas y justas". No hace distinción entre empleo formal o informal. El Estado debe garantizar unos mínimos para protegerlos. Por esta razón, entre otras previamente expuestas, en Colombia se tiene definido que la Seguridad Social es un derecho irrenunciable de todo ciudadano. El Ministerio de Salud y Protección Social considera que el trabajo no puede estar aislado de la dignidad humana y, por ende, la responsabilidad del Estado "va más allá de garantizar el derecho a tener un trabajo (...), sino que el empleado se encuentre en condiciones de seguridad, tranquilidad, estabilidad, igualdad, remuneración justa". Estos elementos llevan a sustentar un trabajo digno que "garantice al trabajador la satisfacción más plena posible de todos sus derechos". El garantizar estas condiciones para lograr un trabajo decente es el medio adecuado para "lograr un desarrollo equitativo, inclusivo y sostenible" (Ministerio de Salud y Protección Social, n. d.).

Sin embargo, en el ámbito nacional se observa otro panorama de gran desigualdad. Según el seguimiento estadístico realizado por el DANE respecto al empleo formal e informal en el área urbana en las 23 principales áreas metropolitanas entre 2001 y 2010, la informalidad ha representado aproximadamente un 50\%, situación que no ha cambiado sustancialmente en el informe de la población trabajadora del sector informal del trimestre noviembre 2014-enero 2015 (DANE, 2015), que fue de $49.3 \%$, similar al mismo trimestre del año inmediatamente anterior (DANE, 2014). En este sector existe un importante grupo de trabajadores del sector informal por subsistencia que, a diferencia de los demás trabajadores, realizan una actividad para poder garantizar su mínimo vital (Congreso de la República de Colombia, 2010), no tienen un contrato laboral ni precario, ni de ninguna otra índole.

En este sector de la economía colombiana, la gran mayoría de la población no está bajo la protección del Sistema de Riesgos Laborales, a pesar de que exista una base de principios desde los convenios internacionales y la Constitución Nacional que determina a la Seguridad Social como un derecho fundamental. Esta realidad dista mucho del ideal del aseguramiento al Sistema General de Seguridad Social, pues existe desprotección, principalmente en riesgos laborales y pensión, lo que hace que el empleo informal quede por fuera de las acciones preventivas de la salud ocupacional, hoy llamadas seguridad y salud en el trabajo, incrementando los riesgos laborales a los que están expuestos. En la atención en salud están cubiertos por el régimen subsidiado que, según datos de un estudio local, es aproximadamente del $82 \%$ en trabajadores del sector informal venteros en calles y aceras de la ciudad de Medellín (Garzón Duque et al., 2014). Este sistema ha asimilado la carga de la atención de los eventos adversos generados por el trabajo, sea accidente o enfermedad laboral, que finalmente se traduce en que quienes cotizan al Sistema Seguridad Social en Salud Contributivo son los que están financiando la atención de estos eventos que deberían estar cubiertos por el Sistema de Riesgos Laborales, con 
agravantes como una atención no oportuna y sin los beneficios económicos a los que tendrían derecho si estuvieran vinculados al Sistema de Riesgos Laborales.

Se concluye entonces que estos derechos que están definidos como fundamentales en la Constitución no son necesariamente garantistas del acceso al sistema de riesgos laborales para la población trabajadora del sector informal. Frente a este panorama, el Estado ha desarrollado diferentes normas para dar protección a esta población vulnerable y, así, vincularlos al Sistema de Riesgos Laborales. La Ley 1562 de 2012 permite al trabajador independiente del sector informal afiliarse al Sistema de Riesgos Laborales siempre y cuando cotice al régimen contributivo en salud, sin otras condicionantes, lo que abre la posibilidad de vinculación a una ARL, pero no se tiene información del porcentaje de afiliaciones que se han logrado con esta medida, aunque se puede deducir que es minoritaria, sea por las dificultades económicas de asumir el pago de las cotizaciones a salud y a riesgos laborales por parte del trabajador informal o independiente, como por las dificultades que presentan las ARL para su afiliación. En efecto, las ARL pueden argumentar lo que reglamenta el Decreto 723 de 2013 sobre la afiliación al Sistema de Riesgos Laborales, y exigir contratos formales de trabajo superiores a un mes o que sean trabajadores independientes que realizan actividades catalogadas como de alto riesgo, lo que es una verdad a medias, como ya se ha expuesto previamente. Teniendo en cuenta estas dos normas, se puede observar una gran posibilidad para la población trabajadora informal e independiente para afiliarse al Sistema de Riesgos Laborales, bajo la Ley 1562 de 2012. Igualmente, el Decreto 2616 de 2013 permite el acceso de los trabajadores del sector informal al sistema de riesgos laborales y a pensión, bajo la consideración de que "un sector importante de trabajadores dependientes que hoy son informales y que, por devengar menos de un salario mínimo, generalmente no se integran al sistema de seguridad social". Esta norma ha permitido que una gran población trabajadora informal, que es beneficiaria del régimen subsidiado en salud, pueda afiliarse a una ARL y a un Fondo de Pensiones, sin perder su calidad de beneficiarios del régimen subsidiado en salud.

Dando respuesta a la pregunta: ¿el sistema de riesgos laborales en Colombia, realmente ampara al trabajador del sector informal?, los autores consideran que sí, desde una visión ideal, pues se tiene una Constitución que protege el trabajo digno, independiente de si es formal o informal, además, que determina como derecho fundamental la seguridad social que abarca no solo salud, sino también riesgos laborales y pensión. El Estado ha promulgado una serie de normas que han beneficiado a la población trabajadora del sector informal en el ingreso al sistema de riesgos laborales, tales como la Ley 1562 de 2012, que permite en forma voluntaria la afiliación al Sistema de Riesgos Laborales bajo la condición de aportar al Sistema de Salud; el Decreto 723 de 2013 que solo regula la afiliación obligatoria a este Sistema a quienes están vinculados 
con un contrato formal de prestación de servicios superior a un mes o al independiente que labore en actividades catalogadas de alto riesgo, y el Decreto 2616 de 2013 que permite la afiliación a riesgos laborales a quienes laboran con contrato de duración menor a un mes y que devenguen menos de un salario mínimo mensual legal vigente. Pero la realidad de la población informal es otra, pues se caracterizan por tener baja productividad, trabajos precarios y, en no pocas ocasiones, de subsistencia, con baja cobertura en seguridad social en pensiones y riesgos laborales, pues en salud están protegidos por el régimen subsidiado. Por esta misma situación, el empleo informal está por fuera de las acciones preventivas de la salud ocupacional, hoy llamado seguridad y salud en el trabajo, incrementando los riesgos laborales a los que están expuestos, entre otras razones porque no logra identificarlos o porque no sabrán como minimizarlos, llevando a aumentar la prevalencia de enfermedades y accidentes laborales que finalmente se convertirán en una carga para el sistema de salud del régimen subsidiado o contributivo.

\section{BIBLIOGRAFÍA}

Barattini, M. (2009). El trabajo precario en la era de la globalización: ¿Es posible la organización? Polis (Santiago), 8(24), 17-37. http://doi. org/10.4067/S0718-65682009000300002

Bustamante Izquierdo, J. P. (2011, agosto). Los retos de la economía informal en Colombia. Ministerio de Hacienda y Crédito Público. Retrieved from http://www.minhacienda.gov. co/portal/page/portal/HomeMinhacienda/ politicafiscal/reportesmacroeconomicos/ NotasFiscales/Boletin\%209\%20Los\%20 retos\%20de\%20la\%20economia\%20informal\%20en\%20Colombia.pdf.

Colombia. Constitución Política (1991). Retrieved from http://www.secretariasenado.gov.co/ index.php/constitucion-politica

Congreso de la República de Colombia. Ley 100 de 1993 Por la cual se crea el sistema de seguridad social integral y se dictan otras disposiciones, $\S$ Diario Oficial. N. 41.148 (1993). Retrieved from http:// www.alcaldiabogota.gov.co/sisjur/normas/ Normal.jsp?i $=5248$

Congreso de la República de Colombia. Ley 1429. Por la cual se expide la ley de formalización y generación de empleo, § Diario Oficial N. 47.937 (2010). Retrieved from http://www. secretariasenado.gov.co/senado/basedoc/ ley_1429_2010.html

Congreso de la República de Colombia. Ley 1450 de 2011. Por la cual se expide el Plan Nacional de Desarrollo, 2010-2014, § Diario Oficial N. 48.102 (2011). Retrieved from http://www. secretariasenado.gov.co/senado/basedoc/ ley_ 1450 _ 2011.html

Congreso de la República de Colombia. Ley 1562 (11 de julio de 2012) Por la cual se modifica en sistema de riesgos laborales y se dictan otras disposiciones en materia de salud ocupacional, § Diario Oficial N. ${ }^{\circ} 48.488$ (2012). Retrieved from http://www. secretariasenado.gov.co/senado/basedoc/ ley_ 1562 _ 2012.html

Corte Constitucional de Colombia. (1992). Sentencia T 581 de 1992. Retrieved from http:/www.corteconstitucional.gov.co/ relatoria/1992/T-581-92.htm

Corte Constitucional de Colombia. (2004). Sentencia T 875 de 2004. Retrieved from http:// www.corteconstitucional.gov.co/relatoria/2004/t-875-04.htm

Corte Suprema de Justicia. (2005, June 30). Sentencia 22656 de 2005. Retrieved from 
http://190.24.134.69/Sentencias/Laboral/ 2005/Dra.Isaura\%20Vargas\%20D\%C3\%ADaz/ SENTENCIAS/22656(30-06-05).doc

DANE. (2009, December 30). Metodología Informalidad Gran Encuesta Integrada de Hogares -GEIH-. Retrieved from https://www.dane. gov.co/files/investigaciones/boletines/ ech/ech _informalidad/metodologia _ informalidad.pdf

DANE. (2014, March 10). Resumen ejecutivo. Medicion del empleo informal y seguridad social. Trimestre móvil noviembre 2013 enero 2014. Departamento Administrativo Nacional de Estadística -DANE-. Retrieved from https://www.dane.gov.co/files/investigaciones/boletines/ech/ech _ informalidad/ re _ech _ informalidad_nov _ ene2014.pdf

DANE. (2015, March 10). Resumen ejecutivo. Medición del empleo informal y Seguridad Social. Trimestre móvil noviembre de 2014 a enero de 2015. Departamento Administrativo Nacional de Estadística -DANE-. Retrieved from http://www.dane.gov.co/ files/investigaciones/boletines/ech/ech/ Res_ejecutivo _ Informalidad _ Nov14_en e15.pdf

Dirección Seccional de Salud de Antioquia. (2013). Análisis de la Situación Salud del Departamento de Antioquia 2012. Retrieved May 25, 2015, from http://www.dssa.gov.co/ minisitio-dssa/index.php/diagnostico-dela-situacion-salud/salud-y-ambito-laboral

Farné, S., Vergara, C. A., E Baquero, N. (2011). La calidad del empleo en medio de la flexibilización laboral. Colombia 2002-2010. Universidad Externado de Colombia. Retrieved from http://portal.uexternado.edu.co/pdf/6 derechoSeguridadSocial/observatorio/publicaciones/otras/calidad-empleo-2011.pdf

Flórez, C. E. (2002). The Function of the Urban Informal Sector in Employment: Evidence from Colombia 1984-2000 (DOCUMENTOS CEDE No. 003595). Universidad de los Andes-CEDE.
Retrieved from https://ideas.repec.org/p/ col/000089/003595.html

Garzón Duque, M. O., Gómez Arias, R. D., E Rodríguez Ospina, F. L. (2014). Indicadores y condiciones de salud en un grupo de trabajadores informales" venteros" del centro de Medellín Colombia) 2008-2009. Investigaciones Andina, 16(28), 932-948.

Gómez Peñaloza, I. C. (2014). Aciertos y desaciertos normativos del sistema de riesgos laborales de los trabajadores independientes en Colombia. Diálogos de Derecho y Política, 0(13). Retrieved from http://aprendeenlinea.udea. edu.co/revistas/index.php/derypol/article/ view/19905

Guataquí R, J. C., García S, A. F., \& Rodríguez A. M. (2011). El perfil de la informalidad laboral en Colombia. Perfil de Coyuntura Económica, 0 (16), 91-115.

Jaramillo, F., Gómez, M., E García-Suaza, A. (2013). Efecto de la política fiscal en un modelo de equilibrio general dinámico con sector informal: una aplicación para Colombia. Serie Documentos de Trabajo, N. ${ }^{\circ}$ 139. Retrieved from http://www.urosario.edu.co/urosario _ files/ bd/bdlf1a25-el22-4561-8c5f-d872e40a6ea0. pdf

Levaggi, V. (2004, August 9). ¿Qué es el trabajo decente? [Noticia]. Retrieved June 9, 2015, from http://www.ilo.org/americas/sala-deprensa/WCMS_LIM_ 653 _ SP/lang- es/ index.htm

Mendoza Martelo, G. E. (2010). La jurisprudencia de la Corte Constitucional frente a la creación del trabajo formal. Presented at the $6 .^{\circ}$ encuentro de la jurisdicción constitucional. "Diálogos con la Nación," Bogotá. Retrieved from http://www.corteconstitucional.gov.co/ viencuentro/conferencia\%20gemm.php 
Ministerio de la Protección Social. Decreto 3039 de 2007 Por el cual se adopta el Plan Nacional de Salud Pública 2007-2010., § Diario Oficial. N. 46.716 (2007). Retrieved from http://www. minsalud.gov.co/Normatividad_Nuevo/ DECRETO\%203039\%20DE\%202007.PDF

Ministerio del Trabajo. (2012, September 25). Trabajadores informales tendrán cobertura de riesgos laborales. Retrieved May 25, 2015, from http://www.mintrabajo.gov.co/ medios-septiembre-2012/1076-trabajadoresinformales-tendran-cobertura-de-riesgoslaborales.html

Ministerio del Trabajo. Decreto 2616 de 2013 Por medio del cual se regula la cotización a seguridad social para trabajadores dependientes que laboran por períodos inferiores a un mes, se desarrolla el mecanismo financiero y operativo de que trata el artículo 172 de la Ley 1450 de 2011 y se dictan disposiciones tendientes a lograr la formalización laboral de los trabajadores informales, § Diario oficial. N. ${ }^{\circ} 48.980$ (2013). Retrieved from http://www.mintrabajo.gov.co/normatividad/ decretos/421-noviembre-2013/2616-decreto2616-del-20-de-noviembre-de-2013.html

Ministerio del Trabajo. (2014, July 3). Cartilla de riesgos laborales para trabajadores. Retrieved from http://www.mintrabajo.gov. co/medios-febrero-2014/3090-cartilla-deriesgos-laborales-para-trabajadores.html

Ministerio de Salud y Protección Social. (2012). Plan Decenal de Salud Pública. Retrieved from http://www.minsalud.gov.co/plandecenal/ Paginas/home2013.aspx

Ministerio de Salud y Protección Social. Resolución 1841 de 2013. Plan Decenal de Salud Pública 2012-2021. Dimensión salud y ámbito laboral., § Diario Oficial. N. 48.811 (2013). Retrieved from http://www.minsalud.gov.co/ plandecenal/Paginas/home2013.aspx

Ministerio de Salud y Protección Social. (2013b, August). Informe de actividades 2012-2013.
Sector Administrativo de Salud y Protección Social. Retrieved from http://www.minsalud. gov.co/Documentos\%20y\%20Publicaciones/ INFORME\%20AL\%20CONGRESO\%2020122013.pdf

Ministerio de Salud y Protección Social (n. d.). Del trabajo y derecho del trabajo. Retrieved from http://www.ut.edu.co/administrativos/images/DOCUMENTOS\%20ADMINISTRATIVOS/ OBSERVATORIOS/Del _ Empleo _ y _ Recursos _ Humanos/Legislaci\%C3\%B3n_laboral _ documentos/derecho _ al _ trabajo.pdf

Ministerio de Trabajo. Decreto 1295 de 1994. Por el cual se determina la organización y administración del Sistema General de Riesgos Profesionales, $\S$ Diario Oficial N. ${ }^{\circ}$ 41.405 (1994). Retrieved from http://www. secretariasenado.gov.co/senado/basedoc/ decreto _ 1295 _ 1994.html

Ministerio de Trabajo. Decreto 1607 de 2002. Por el cual se modifica la Tabla de Clasificación de Actividades Económicas para el Sistema General de Riesgos Profesionales y se dictan otras disposiciones, $\S$ Diario Oficial N. ${ }^{\circ} 44.892$ (2002). Retrieved from http:// jacevedo.imprenta.gov.co/tempDownloads/ 44D8921440861207655.pdf

Ministerio de Trabajo. Decreto 0723 de 2013. Por el cual se reglamenta la afiliación al Sistema General de Riesgos Laborales de las personas vinculadas a través de un contrato formal de prestación de servicios con entidades o instituciones públicas o privadas y de los trabajadores independientes que laboren en actividades de alto riesgo y se dictan otras disposiciones., $\S$ Diario Oficial. N. ${ }^{\circ} 48762$ (2013). Retrieved from http://www.mintrabajo.gov.co/normatividad/decretos/415mayo-2013/1830-decreto-0723-del-15-deabril-de-2013.html

Ministerio de Trabajo. Decreto 1443 de 2014 Por el cual se dictan disposiciones para la implementación del Sistema de Gestión de 
la Seguridad y Salud en el Trabajo (SG-SST), $\S$ Diario Oficial. N. ${ }^{\circ} 49.229$ (2014). Retrieved from http://www.mintrabajo.gov.co/normatividad-julio-decretos-2014/3700 -decreto1443-del-31-de-julio-de-2014.html

Núñez Castrejón, A., \& Gómez Chiñas, C. (2008). Controversia y debate actual sobre el sector informal. Análisis Económico. Retrieved from http://www.redalyc.org/resumen. oa?id=41311483007

Núñez Méndez, J. A. (2002, October 29). Empleo informal y evasión fiscal en Colombia. Departamento Nacional de Planeación, Dirección de Estudios Económicos. Archivo de economía, documento 210. Retrieved from https:// colaboracion.dnp.gov.co/CDT/Estudios\%20 Econmicos/210.pdf

OIT. (1993a, January). Resolución sobre la Clasificación Internacional de la Situación en el Empleo (CISE). Retrieved June 15, 2015, from http://www.ilo.org/global/statistics-anddatabases/standards-and-guidelines/resolutions-adopted-by-international-conferences-of-labour-statisticians/WCMS _ 087564/ lang- es/index.htm

OIT. (1993b, January 1). Resolución sobre las estadísticas del empleo en el sector informal [Resolución]. Retrieved June 15, 2015, from http://www.ilo.org/global/statistics-anddatabases/standards-and-guidelines/resolutions-adopted-by-international-conferen-
ces-of-labour-statisticians/WCMS _ 087486/ lang- es/index.htm

OIT. (1996). Misión y objetivos. Retrieved May 25, 2015, from http://ilo.org/global/about-the-ilo/ mission-and-objectives/lang--es/index.htm

OIT. (2003). Decimoséptima Conferencia Internacional de Estadísticos del Trabajo. Ginebra, 24 de noviembre a 3 de diciembre de 2003: OIT. Retrieved from http://www.ilo.org/wcmsp5/ groups/public/ - dgreports/ - stat/documents/meetingdocument/wcms _ 087570. pdf

ONU. (1966, December 16). Pacto Internacional de Derechos Económicos, Sociales y Culturales. Retrieved from http://www.ohchr.org/SP/ ProfessionalInterest/Pages/CESCR.aspx

ONU. (n.d.). Declaración Universal de Derechos Humanos. Retrieved from http://www.un.org/ es/documents/udhr/

Portafolio. (2011, June 7). Hay 13 millones de personas sin protección laboral. Retrieved June 15, 2015, from http://www.portafolio. co/economia/hay-13-millones-personasproteccion-laboral

Vásquez-Trespalacios, E. M., E Martínez-Herrera, E. (2013). Políticas públicas en seguridad social para la protección de los trabajadores informales en Colombia. Gerencia y Políticas de Salud, 12(24). http://doi.org/10.11144/6094 
